\title{
Rapid generation of secondary fibroblasts through teratoma formation
}

\author{
Sang Mi Cho ${ }^{* *}$, Jung Sun Park ${ }^{1 *}$, Byungkuk Min ${ }^{1,2}$, Sujin Kwon¹, and Yong-Kook Kang ${ }^{1,2}$ \\ ${ }^{1}$ Development and Differentiation Research Center, KRIBB, South Korea and ${ }^{2}$ Department of Functional \\ Genomics, University of Science and Technology (UST), Daejeon, South Korea
}

*S.M.C. and J.S.P. contributed equally to this work

BioTechniques 59:34-41 (July 2015) doi 10.2144/000114309

Keywords: teratoma; induced pluripotent stem cell (iPSC); reprogramming; secondary fibroblast; doxycycline; mouse embryonic fibroblast (MEF); stem cell

The use of secondary or reprogrammable cells in the production of induced pluripotent stem cells (iPSCs) circumvents random infection by various viral particles and random, uncontrollable integrations of the viral genomes into different genomic loci. We have developed a convenient method for repeatedly producing genetically identical secondary fibroblasts via teratoma formation using pre-existing iPSCs. The iPSCs used in this study carried doxycycline (Dox)-inducible transgenes for four transcription factors in their genome. Teratoma-derived primary cells (TOFs) were obtained in a huge amount during the culture of teratomas and showed good ability to form iPSCs similar to that of regular secondary fibroblasts. Immunohistochemistry analysis demonstrated the potential of TOF-derived iPSCs to differentiate into all three germ layers. The gene expression profiles of these TOFs and their iPSCs closely mimicked those of regular embryonic fibroblasts and embryonic stem cells/iPSCs, respectively. The possibility that the iPSCs were derived from a small part of pluripotent cells lurking in the TOF population was precluded by the observation of doxycycline-dependent and PluriSin (a compound selectively eliminating pluripotent cells)independent formations of iPSCs. Our results showed that the TOFs retained the capability to mediate cellular reprogramming, similar to that of regular secondary fibroblasts.

Mouse and human fibroblasts can be reprogrammed into induced pluripotent stem cells (iPSCs) through the viral introduction of four transcription factors: Oct4, Sox2, Klf4, and c-Myc (1-6). This approach can cause random infections of target cells with different numbers of viral particles and random integration of the viral genomes into different genomic loci that are uncontrollable by any means. The resulting genetic heterogeneity complicates the exploration and interpretation of crucial molecular events that govern somatic cell reprogramming. Thus, secondary repro- gramming systems were developed to circumvent the need for virus-mediated transduction and to reduce the heterogeneity of reprogramming factor-expressing cells (7-11). All of the cells in each secondary system carry the same doxycycline (Dox)inducible transgenes. These genetically homogeneous cells, therefore, could be converted to iPSCs upon simple exposure to Dox without additional viral infection.

Among these secondary systems, the so-called secondary mouse embryonic fibroblast (secondary MEF) system has drawn great attention (10). This system relies on cells that can form embryonic stem cell (ESC)-like colonies within 4 days after Dox exposure, and this process is much faster than that of 4F2A cells, another secondary cell system obtained from a single-gene transgenic mouse, where initial colonies normally appear 16 days after Dox exposure (12). This advantage is of great importance for the study of the molecular mechanisms of reprogramming, because it allows the researchers to save a large amount of time on sample collection. These MEFs are collected from a chimeric fetus produced by blastocyst injection of

\section{METHOD SUMMARY}

Here, we present a method for repeatedly producing genetically identical secondary fibroblasts via teratoma formation using pre-existing iPSCs carrying doxycycline (Dox)-inducible transgenes for four transcription factors in their genome. Our approach comprises three steps: (i) teratoma formation by iPSC injection into SCID mice, (ii) secondary fibroblast collection from teratomas, and (iii) iPSC derivation from secondary fibroblasts by Dox treatment. This method bypasses the use of demanding transgenic techniques and allows the mass-production of secondary fibroblasts in a feasible way. 
primary iPSCs (10). Although this protocol has shown great promise for reprogramming, it requires the use of a series of challenging transgenic technologies, including embryo manipulation and embryo transfer to the uterus of the recipient mouse. The technical barriers associated with this method limit its use in most laboratories. Here, we present a protocol for generating secondary fibroblasts through the formation of a teratoma in severe combined immunodeficiency (SCID) mice. This method bypasses the use of demanding transgenic techniques and allows the massproduction of secondary fibroblasts in a more feasible way. This teratoma-mediated method for producing secondary fibroblasts has shown good potential to facilitate the study of epigenetic reprogramming mechanisms, and it will serve as a powerful tool for the development and optimization of iPSC production strategies.

\section{Materials and methods}

\section{Ethics statement}

This study was carried out in strict accordance with the recommendations in the Guide for the Care and Use of Laboratory Animals of the National Livestock Research Institute of Korea. The protocol was approved by the Committee on the Ethics of Animal Experiments of the Korea Research Institute of Bioscience and Biotechnology. All surgery was performed under sodium pentobarbital anesthesia, and all efforts were made to minimize suffering.

Chimera formation and secondary MEF isolation

The NGFP1 iPSC line (10) was purchased from Stemgent Inc. (Cambridge, MA). NGFP1 iPSCs were injected into BDF1 (C57) B6 $\times$ DBA/1) hybrid blastocysts 94-98 h after hCG injection. A flat-tip microinjection pipette with an internal diameter of 12-15 $\mathrm{mm}$ was used for iPSC injection. About 10 cells were placed into the blastocyst cavity, and the injected blastocysts were immediately transferred to recipient females. To construct chimeric fetuses carrying cells stemmed from teratoma-derived iPSCs, tertiary iPSCs (see below) were transfected with pDsRed2-C1 (Takara Clontech, Otsu, Japan) plasmids using Nucleofector (Lonza, Basel, Switzerland), and those iPSC colonies that survived drug selection and contained red fluorescence signal were pooled before use in blastocyst injection. Twelve to 15 blastocysts were
Table 1. Primer sequence information.

\begin{tabular}{|c|c|c|c|}
\hline Gene & Primer seque & nce ( $5^{\prime}$ to $\left.3^{\prime}\right)$ & Accession \# \\
\hline Oct4 & CCCAGGTCCCCACTTTGGCAC & АCCCCTGTTGTGCTTTTAATCCC & NM_001252452.1 \\
\hline Nanog & CACCAGTGGAGTATCCCAGCAT & TGGCAGAGAAGTTTTGCTGCAAC & NM_028016.2 \\
\hline Lin28a & GGAATCTTGGGCTTCATTTGT & TTAGCTATCAACCATCACGCT & NM_145833.1 \\
\hline Sox2 & CCATGTATAGATCTGGAGGAAAA & CAAATATTAAAACTTTTTTTTCATCG & NM_011443.3 \\
\hline Dppa3 & TTGAGCAGAGACAAAAAAGG & TAATTCTTCCCGATTTTCGC & NM_139218.1 \\
\hline Dppa4 & TCCTGGGAACTCGCGTGGTT & TTGGGGCACAGCATGTTGTC & NM_028610.2 \\
\hline Dppa5a & GAAGAACTTATCGAGGTCTT & GACTGGAAACTGGCTTCACT & NM_025274.3 \\
\hline Dnmt3l & TGCTGTTCCGGGGGTACCCT & AGGCTCAGTACCCGCACTGG & NM_019448.3 \\
\hline Fbxo15 & TCTGTGTAAATCCTGGCCTC & CACAAATGTAGTGTCGGGAG & NM_015798.3 \\
\hline Zfp42 & CATCCTAACCCACGCAAAGG & AAGAGTTGCAAAATTATTTT & NM_009556.3 \\
\hline Tcf3 & ATGCCTTAACTATGTAAGAC & GACATGAGACTTTTATACAA & NM_011548.4 \\
\hline Sall4 & ТTTTTCTTAАСССАССТССТ & TTTGGTTTGTTTTTGTTGTT & NM_175303.3 \\
\hline Triml1 & TCAGGCCATCCTGAACCATG & CGGATTTGCTGGGTCAGCTG & NM_177742.4 \\
\hline Prdm 14 & GCAATTTGCACTACCTAGAG & AGTTAGACATCAACAAGAAT & NM_001081209.2 \\
\hline Fgf4 & TTTGTGCTGGAACTCCATAAT & GAAAAATATAAGGGTTACTTGA & NM_010202.5 \\
\hline KIf4 & GGTTTATTTCTGTAACATTTTTATG & TTAAAGGCATACTTGGGAATAAAC & NM_010637.3 \\
\hline KIf5 & CCGCTACATTGCTTCCAAAC & TTCTCCAGATCCGGGTTACT & NM_009769.4 \\
\hline$c-m y c$ & AAAAACAATTAAATACAAATCTGTTA & CTCGTGAGAGTAAGGAGAACGG & NM_010849.4 \\
\hline Col1a2 & TGGCCCATCTGGTAAAGAAG & ACCTTTGCCACCTTGAACAC & NM_007743.2 \\
\hline$A x I$ & GAGGAAGAAGGAGACTCGAT & TCCTTCAGCTCTTCACTGAT & NM_009465.4 \\
\hline Col4a2 & CTCTCCCCATGTAGGACATA & CCAGGTCTAGACAGAAGCAC & NM_009932.3 \\
\hline Prx1 & CCGGGAATTTAGCGTTTGTA & САTCCСАСТTCСТCСТTTGA & NM_011127.2 \\
\hline P4hb & GAGGACAACGTCCTGGTGTT & GCCTTCTGCCTTCAGTTTTG & NM_011032.2 \\
\hline Fsp 1 & AGGCCCTGGATGTAATTGTG & GCTGTCCAAGTTGCTCATCA & NM_011311.2 \\
\hline Fap & TCAACTGTGATGGCAAGAGC & CATGGTTCTGGTCAGAGTAC & NM_007986.3 \\
\hline Vim & AGCCACGCTTTCATACTGCT & ATGCTTCTCTGGCACGTCTT & NM_011701.4 \\
\hline Gapdh & CCAGTATGACTCCACTCACG & TTCACACCCATCACAAACAT & NM_008084.2 \\
\hline
\end{tabular}

transferred to the uterine horn of a pseudopregnant BDF1 mouse at 2.5 days post coitum. Secondary MEFs were isolated as described previously (10). Briefly, chimeric embryos were collected at E13.5, and the head and internal organs were removed. The remaining carcass was physically dissociated and incubated in trypsin at $37^{\circ} \mathrm{C}$ for $20 \mathrm{~min}$. Dissociated cells were resuspended in MEF media containing $2 \mu \mathrm{g} / \mathrm{mL}$ puromycin and expanded for 2 passages before freezing or plating on Dox-containing medium for reprogramming experiments.

Reprogramming and colony picking Unless indicated otherwise, all cell culture materials were purchased from Gibco/ Life Technologies (Grand Island, NY). For reprogramming, as described previously (13), secondary MEFs or (teratomaderived primary cells) TOFs at passage 3 (p3) were plated on Matrigel (BD Biosciences, San Jose, CA)-coated dishes at $2.0 \times 10^{4}$ cells $/ \mathrm{cm}^{2}$ in MEF medium [DMEM supplemented with 10\% FBS, 0.1 $\mathrm{mM}$ nonessential amino acids (NEAA), and $2 \mathrm{mM}$ Glutamax]. The following day, culture medium was replaced with fresh MEF medium containing $2 \mu \mathrm{g} / \mathrm{mL}$ Dox (Bio Basic, Markham, Ontario, Canada), in which cells were cultured another $24 \mathrm{~h}$. Medium was changed again with Dox-containing iPS medium (knockout DMEM supplemented with 10\% knockout serum replacer, 5\% ES-qualified FBS, 0.1 mM NEAA, 2 mM Glutamax, and 0.055 $\mathrm{mM} \beta$-mercaptoethanol), and the culture was maintained for the indicated periods with medium replaced at every second day with fresh Dox-containing iPS medium. For colony counting, iPSC colonies were first stained for alkaline phosphatase (AP) using the ALSTEM (Richmond, CA) AP staining kit according to the manufacturer's manual. Then, the number of AP-positive colonies was assessed using the colony counting software OpenCFU, which automatically recognizes and counts colonies that meet fixed criteria (13).

\section{RT-PCR}

Total RNA was prepared from cultured cells using the RNeasy mini kit (Qiagen, Hilden, Germany). cDNA was synthesized using an oligo-dT primer and SuperScript III reverse 
A
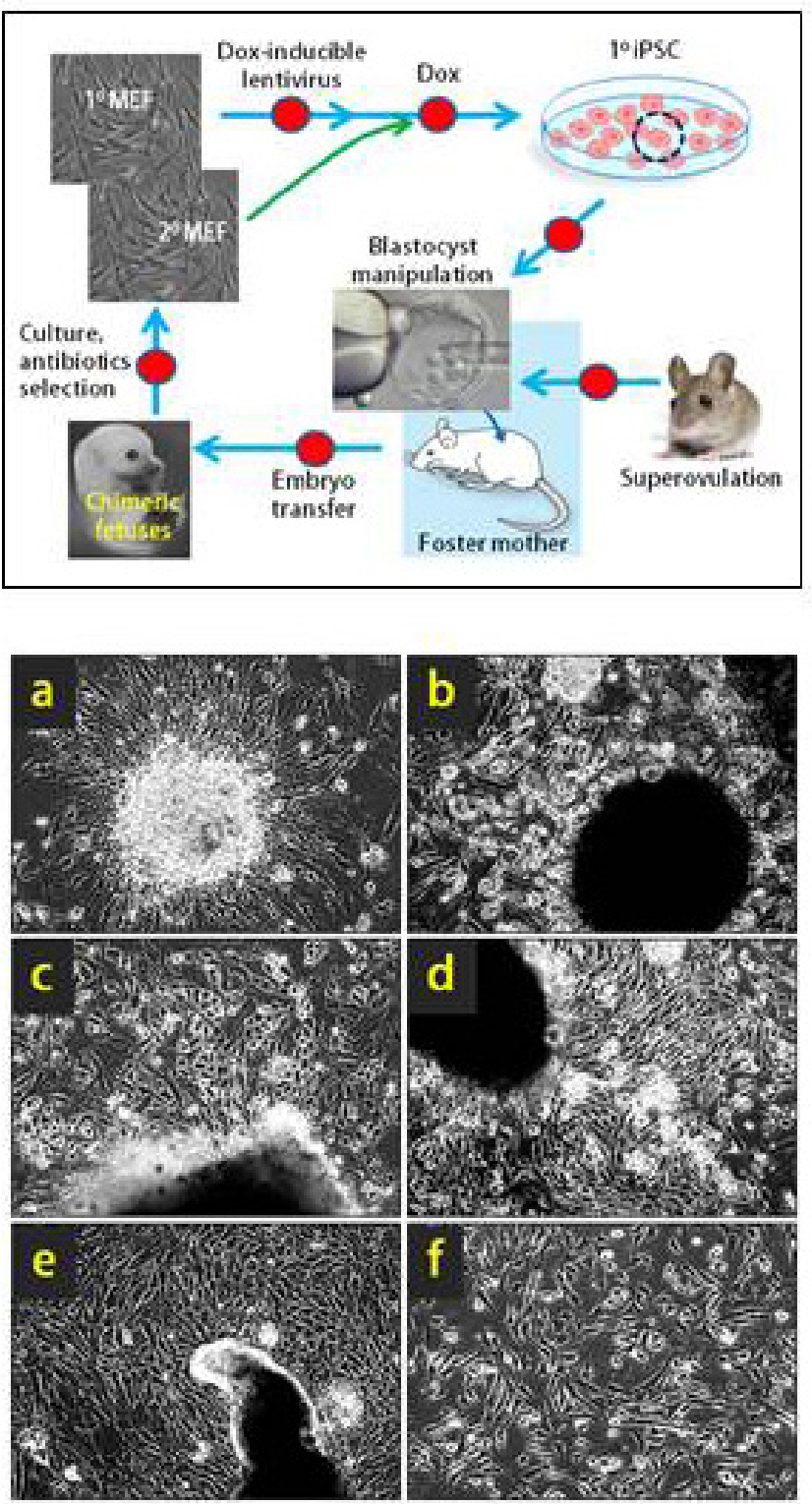

B

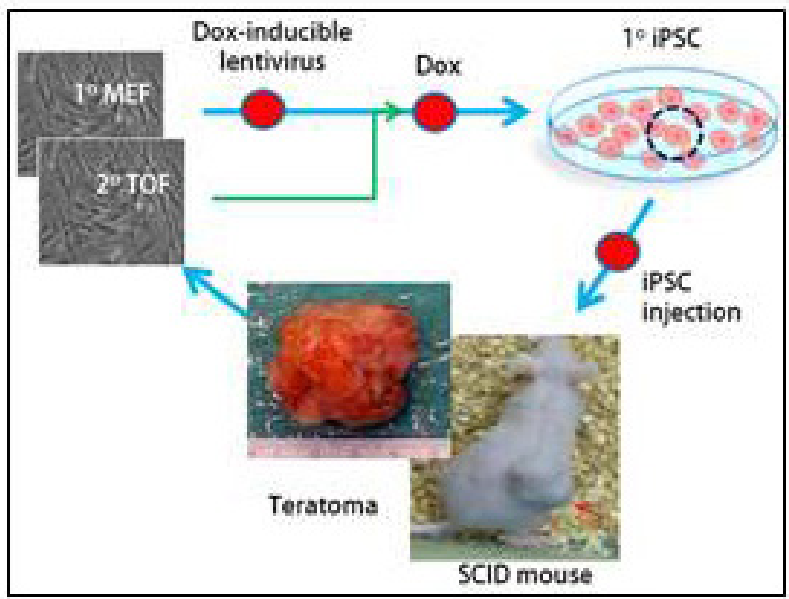

D
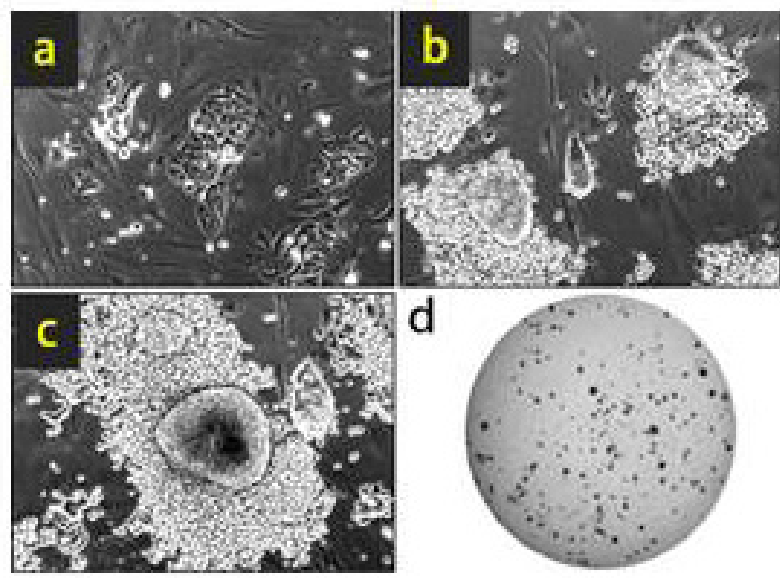

Figure 1. Derivation of secondary fibroblasts from teratomas produced from primary induced pluripotent stem cells (iPSCs). Diagrams illustrating the derivation of secondary fibroblasts using (A) transgenic technology and (B) the teratoma-mediated protocol. (C) Various types of primary cells spread out of the teratoma pieces. (D) Progressive formation of pre-iPSC colonies from teratoma-derived secondary fibroblasts at day 4 (a) and days $6-8(b, c)$ after exposure to doxycycline; and alkaline phosphatase (AP)-positive iPSC colonies observed on days 9-10 (d). transcriptase (Invitrogen, Carlsbad, CA) according to the manufacturer's instructions. Two micrograms of total RNA were used for cDNA synthesis. Primers are listed in Table 1. Reverse transcription was done at $50^{\circ} \mathrm{C}$ for $30 \mathrm{~min}$ in a thermal cycler (DNA engine; BioRad, Hercules, CA) and then PCR was performed using the resultant cDNA with 15 cycles of $94^{\circ} \mathrm{C}$ for $30 \mathrm{~s}, 56^{\circ} \mathrm{C}$ for $30 \mathrm{~s}, 72^{\circ} \mathrm{C}$ for $30 \mathrm{~s}$.

Teratoma formation and collection of teratoma-derived fibroblasts

In order to generate teratomas, $5 \times 10^{6}$ iPSCs were injected subcutaneously into the flanks of recipient SCID mice. Briefly, cells were washed once with PBS and harvested. The cell suspension was then collected and spun down. The cell pellet was resuspended by stepwise addition of a 1:1 mixture of PBS:Matrigel to a final total volume of $200 \mu \mathrm{l}$. Three to 4 weeks later, tumors were surgically isolated and washed with PBS. The masses were sent for immunohistological analysis or minced using a scalpel in $5 \mathrm{~mL}$ of MEF medium for preparation of TOFs. All of the tissue clumps were transferred to a new culture dish and incubated overnight. The next day, an additional $5 \mathrm{~mL}$ MEF medium was added, and the culture was incubated until TOF cells spread out from the bottom of the tissue clumps. Cultures were expanded for two passages before freezing or plating on Dox-containing medium for reprogramming experiments.
Immunostaining and SA- $\beta$-gal analysis iPSC colonies derived from secondary MEF or TOFs were plated on a Matrigel-coated chamber slide without feeder cells for immunostaining, as described elsewhere $(14,15)$. After overnight culture, cells were fixed in 4\% (w/v) formaldehyde for $20 \mathrm{~min}$ at room temperature (RT) and washed in PBS containing 0.05\% Tween 20 (PBST; Fisher Scientific, Ottawa, Ontario, Canada). After permeabilization for 30 min in PBS containing 1\% Triton X-100 (MP Biomedicals, Irvine, CA), samples were blocked in $2 \%$ BSA-PBS (Sigma Aldrich, St. Louis, $\mathrm{MO}$ ) for 30 min at RT and incubated with $\alpha$-Oct4 (sc-8628; Santa Cruz, CA) or $\alpha$-Sox2 (MAB2018; R\&D Systems, Minneapolis, MN) primary antibodies on a slow 
rocker overnight at $4^{\circ} \mathrm{C}$. Alexa Flour 488 or 594-conjugated secondary antibodies (1:300, Molecular Probes, Eugene, OR) were applied for $25 \mathrm{~min}$ at RT in a lightprotective environment. Samples were observed with a Carl Zeiss (Göttingen, Germany) Axiovert 200M fluorescence microscope. Images were captured digitally and merged using Axiovision (v4.7) or Adobe Photoshop software (v7.0). For SA- $\beta$-gal staining, cells were washed 3 times with PBS, fixed with 4\% formaldehyde for 10 min at RT, and washed again twice with PBS. Cells were incubated with a staining solution $(1 \mathrm{mg} / \mathrm{mL}$ X-gal, $5 \mathrm{mM}$ potassium ferricyanide, $5 \mathrm{mM}$ potassium ferrocyanide, $40 \mathrm{mM}$ citric/sodium buffer, $\mathrm{pH}$ 6.0, $150 \mathrm{mM} \mathrm{NaCl}$, and $2 \mathrm{mM} \mathrm{MgCl}_{2}$ in distilled water) at $37^{\circ} \mathrm{C}$ overnight. After a brief wash with PBS, stained cells were observed on a light microscope.

\section{Flow cytometry}

Cells were pelleted by centrifugation, resuspended in PBS containing $0.5 \%$ BSA to a final density of $1 \times 10^{6} \mathrm{cells} / \mathrm{mL}$, and filtered through a nylon membrane to remove cell aggregates. Flow cytometry and cell sorting for GFP fluorescence were performed using a FACStar Plus (Becton Dickinson,
San Jose, CA) with an INNOVA 70-4 argon laser tuned to $488 \mathrm{~nm}$. A minimum of 10,000 events were collected for each analysis. GFP signals were detected with a 530/30-nm bandpass filter.

\section{Results and discussion}

As in the previous study (10), we injected NGFP1 iPSCs into mouse blastocysts, transferred the manipulated embryos into recipient mice, and finally collected the resulting chimeric fetuses at embryonic day 13.5 (E13.5) to obtain secondary MEFs. The genetically homogeneous secondary MEFs that carried Dox-inducible proviral DNAs in the genome were obtained from the chimeric fetuses and enriched by puromycin selection (Figure 1A). Upon exposure to Dox, secondary iPSCs were obtained from these secondary MEFs. Overall, this transgenic procedure is technically challenging, and the puromycin selection process requires a long-term culture in vitro, inevitably leading to the early onset of senescence in the selected cell population.

To overcome these disadvantages, we devised a novel protocol for generating secondary MEFs through teratoma formation (Figure 1B). To form teratomas, we injected secondary MEF-derived secondary iPSCs into nonobese diabetic (NOD)-SCID mice and recovered a teratoma $\sim 3$ weeks later. The teratoma was cut into fine pieces to allow the primary cells to spread out during the next 4 days of culture (Figure 1C). The initial cultures of different pieces displayed a high degree of heterogeneity (Figure 1C, a-e) and were eventually occupied by fibroblast-looking cells, which were named as teratomaderived fibroblast-like cells (TOFs) (Figure $1 \mathrm{C}-\mathrm{f}$ and see also Figure 3, C and D). After treatment with Dox, these tertiary TOFs (Figure 3A) experienced the same iPSC transformation process as did secondary MEFs (10) (Figure 1D, a-c), where alkaline phosphatase (AP)-positive iPSC colonies formed in 8-10 days (Figure 1D-d).

The tertiary TOF-derived iPSCs (tertiary iPSCs) were maintained in culture medium and strongly stained for AP, Oct4 and Sox2 after several passages in the absence of Dox (Figure 2A). As the NGFP1 iPSCs carry a green fluorescent protein (GFP) cDNA targeted to the endogenous Nanog locus (10), the GFP signals observed from the tertiary iPSC colonies indicate the derepression of the endogenous

A

B
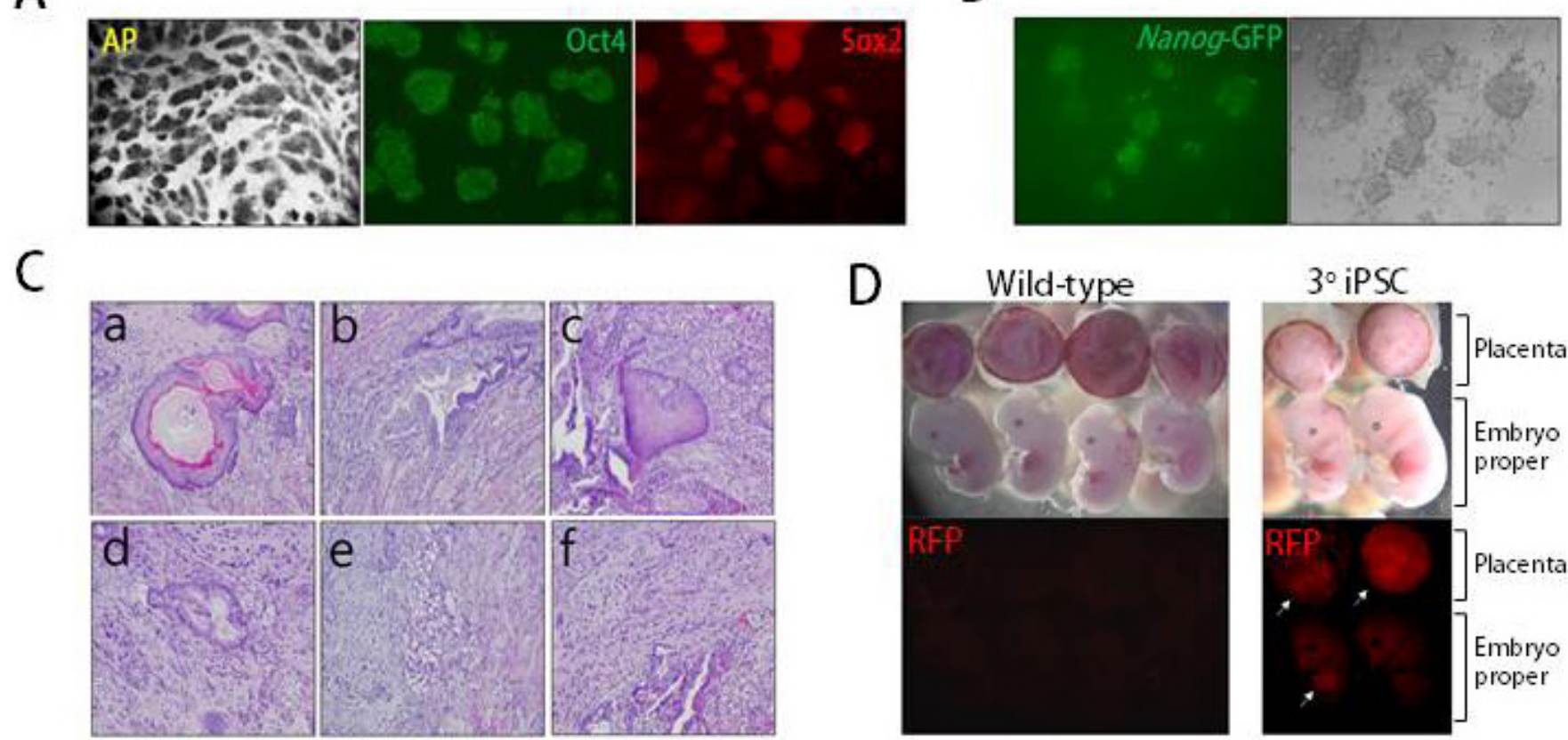

Figure 2. Stem cell features shown in teratoma-derived induced pluripotent stem cells (iPSCs). (A and B) Immunocytochemical analysis of teritiary iPSCs at passage 8 showing positive staining for alkaline phosphatase (AP), Oct4, and Sox2 (A), or green fluorescent protein (GFP) signals expressed by the endogenous Nanog locus (Nanog-GFP) (B). (C) Immunohistological analysis of teratomas established by injecting teratoma-derived fibroblast-like cells (TOF)derived iPSCs into a NOD-SCID mouse. Teratoma sections were stained using hematoxylin and eosin (H\&E), allowing the observation of a squamous cell with a keratin pearl (a), gut-like epithelium (b), bone (c), respiratory epithelium (d), adipocytes (e), and smooth muscle (f). (D) E13.5 chimeric fetuses harboring cells derived from TOF-derived tertiary iPSCs. Tertiary iPSCs stably expressing red fluorescent protein (RFP) were injected into mouse blastocysts to make chimeric fetuses. RFP signals (arrows) are seen both in the placentas and in the embryos proper in $3^{\circ}$ iPSCs chimeric fetuses but not in control fetuses. 
A

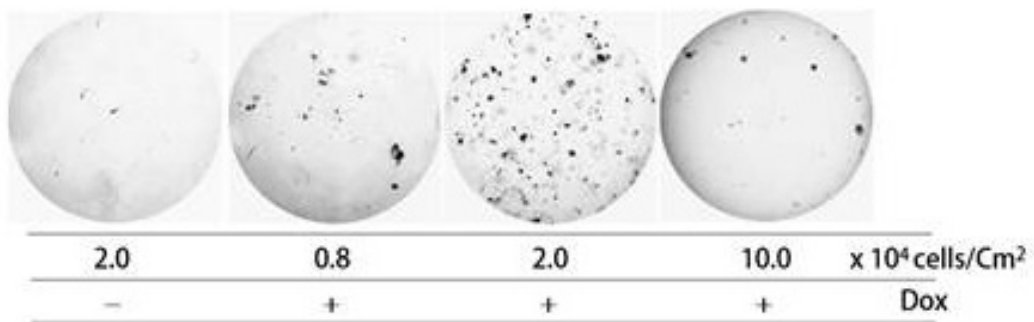

B
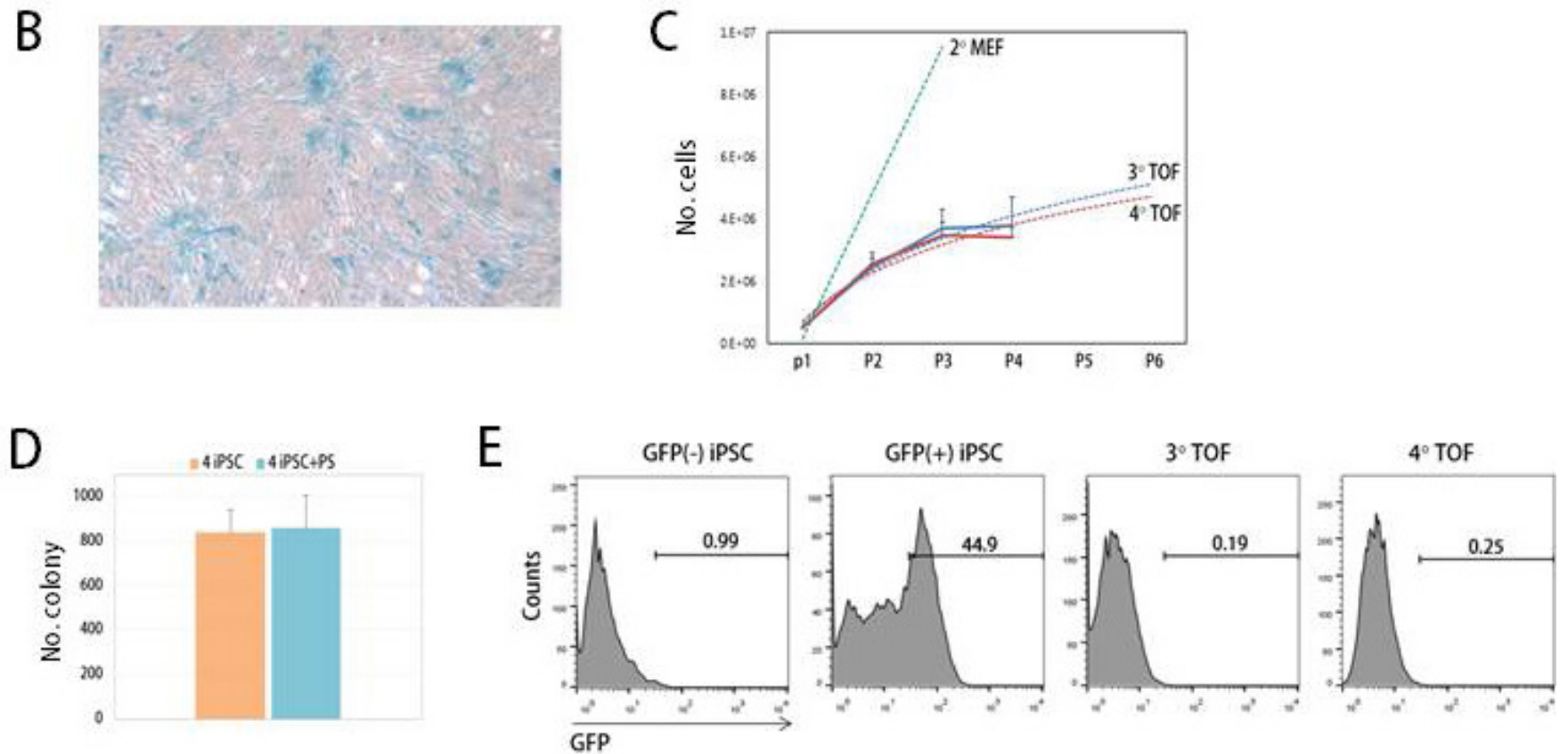

Figure 3. Characterization of teratoma-derived fibroblast like cells (TOFs). (A) Doxycycline (Dox)-dependent and cell density-dependent production of induced pluripotent stem cells (iPSCs) from TOFs. Alkaline phosphatase (AP) staining of pre-iPSC colonies at induction day 10 is shown in a 4-well dish. The number of cells used in each well is indicated. (B) SA- $\beta$-gal analysis. Long-term-cultured TOFs are SA- $\beta$-gal positive (blue), an indication of cellular senescence. (C) Growth curves of TOFs of different generations [tertiary $\left(3^{\circ}\right)$ TOF and quaternary $\left(4^{\circ}\right)$ TOF]. Dotted lines denote predicted trend lines. P1-6: passages 1-6. (D) PluriSin (PS) treatment. PluriSin can remove pluripotent cells from culture. TOFs were pre-treated with $20 \mu \mathrm{M}$ PluriSin for $48 \mathrm{~h}$ before incubation with Dox for $10-11$ days. The number $(859 \pm 150 ; n=8)$ of pre-iPSC colonies derived from PluriSin-treated TOFs was not different ( $P=0.772 ; t$-test) from that derived from control TOFs $(840 \pm 99)$. All experiments were duplicated at least twice. (E) Results of fluorescence-activated cell sorting (FACS). Histograms of fluorescence intensity in the GFP channel are shown for GFPnegative secondary iPSCs derived from 4F2A secondary MEFs (12), GFP-positive secondary iPSCs derived from NGFP1 secondary MEF, tertiary TOF, and quaternary TOF. Numbers in the panels indicate the percentage of GFP-positive cells. These data are representative of triplicate experiments.

Nanog locus (Figure 2B). The results of chromosome counting indicated that $74.5 \%$ $(n=39)$ of the tertiary TOFs showed normal ploidy. Immunohistochemical analysis of the tertiary iPSC-derived teratoma demonstrated the potential of iPSCs to differentiate into three germ layers (Figure 2C). Next, in order to prove the pluripotency of TOF-derived iPSCs, we attempted to make chimeric fetuses by injecting into mouse blastocysts tertiary iPSCs stably expressing red fluorescent protein (RFP). When the resulting fetuses collected at 13.5 days post coitum (dpc) were examined, we were convinced that TOF-derived iPSCs could contribute to both the embryo proper and placenta during development (Figure
2D). Together, these results show that the TOFs and resulting iPSCs retain the same capability for mediating cellular reprogramming as that of the secondary MEFs.

There might be a concern that a tiny amount of cells with a pluripotent character would remain in the TOF population. Several lines of evidence could preclude this possibility. First, TOFs did not form iPSC colonies when cultured in ES medium without doxycycline (Figure 3A). Second, as shown in the derivation of iPSCs from secondary MEFs (10), the formation of iPSCs was highly dependent on the growing density of TOFs (Figure 3A). Third, prolonged culture did not induce TOFs to form iPSC-like colonies and instead made them SA- $\beta$-gal-positive, which could be a sign of cellular senescence (Figure 3B). Fourth, the growth of TOFs was relatively slow compared with secondary MEFs, and they usually ceased to proliferate at around passages 4-5 (Figure 3C). Fifth, the re-injection of TOFs at the second passage could not re-establish teratomas in the SCID mice (data not shown). Sixth, the pre-treatment of TOFs with PluriSin, which served as a pluripotency inhibitor to selectively eliminate pluripotent stem cells (16), did not reduce the number of iPSC colonies ( $P=0.772$, $t$-test; Figure 3D). Finally, we performed FACS analysis. As shown in Figure 3E, while the GFP-positive proportion was $44.9 \%$ in NGFP1 secondary 
MEF-derived iPSCs, the GFP proportions in the tertiary and quaternary TOF cultures were $0.19 \%$ and $0.25 \%$, respectively, which were not different from that in 4F2A secondary MEF-derived iPSCs (12) that do not possess the GFP transgene. Altogether, we conclude that the iPSC colonies induced in this study are generated through the reprogramming of TOFs, rather than by the pre-existing pluripotent cells.

The cyclic procedure of teratoma formation, TOF derivation, and iPSC production was repeated for multiple rounds, as illustrated in Figure 4A. The resulting iPSCs (secondary iPSCs and tertiary iPSCs) and TOFs (tertiary TOFs, and quaternary TOFs) were characterized upon the completion of each cycle. The AP staining results showed that the number of AP-positive colonies was not different among iPSC generations at the $P<0.05$ level, as determined by a one-way analysis of variance (ANOVA) test (Figure 4B). Next, we examined the expression levels in these iPSCs and TOFs at different generations. Two groups of genes that have been reported by many studies to be differentially expressed in mouse ESCs (mESCs) and in fibroblasts were chosen for analysis.
The gene expression profile of tertiary TOF-derived tertiary iPSCs is similar to the profile of secondary iPSCs and that of $\mathrm{J} 1 \mathrm{mESCs}$ (Figure 4C). For the fibroblastspecific genes, tertiary and quaternary TOFs exhibited similar expression profiles to those of ordinary MEFs and secondary MEFs (Figure 4D). These results indicate that the TOFs exhibit a MEF-like gene expression pattern, and TOF-derived iPSCs show mESC- and regular iPSC-like expression patterns, implying that the process of reprogramming teratoma-derived TOFs into iPSCs is as efficient as that using the regular secondary MEFs. Meanwhile, the gene expression result does not preclude the possibility of the presence of other types of cells in TOF culture, so it may be necessary to further characterize the TOF culture by, for example, flow cytometry, for several selected markers.

In this study, we developed a method for repeatedly producing clonally derived, genetically identical secondary fibroblasts by means of teratoma formation using pre-existing Dox-inducible iPSCs. Starting with pre-existing primary iPSCs, this procedure is executed by following three consecutive steps as follows: (i) teratoma formation, (ii) TOF collection from teratoma, and (iii) iPSC derivation from the TOFs by Dox induction. This simple and convenient method enables ordinary laboratories to avoid the use of hard-to-access transgenic technology and obtain iPSCs in large amounts with a short turnaround time. We hope that by eliminating uncontrollable experimental variables, our method could be used as a reliable research tool for iPSC study and that the research platform established based on this technique would be advantageous for studying the mechanism of cell dedifferentiation and for genetic and chemical screening to improve reprogramming efficiency. Hockmeyer et al. (11) previously reported the derivation of secondary fibroblasts from iPSC-derived teratomas but they did not characterize those teratoma iPSCs, nor did theydescribe the derivation protocol. In contrast, we have provided a complete description of the procedure for deriving secondary fibroblasts from iPSC-derived teratomas and a full characterization of the TOF-derived iPSCs.

There is another way of generating secondary fibroblasts. If we had transgenic mice carrying the Dox-inducible

\section{Inluminating Solutions For Your Life science Research!}

Life science and laboratory research professionals depend on high-quality Spectroline ${ }^{\circledR}$ ultraviolet products to make their work faster and easier. Our products are built to exacting standards, ensuring years of reliable, trouble-free service.

\section{UV Crosslinkers}

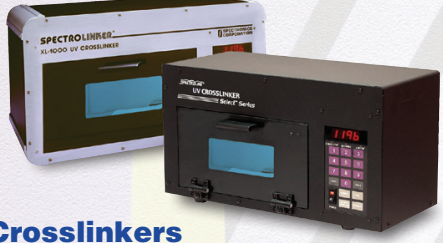

Provide accurate, error-free and super-fast

membrane crosslinking and sample UV dosing

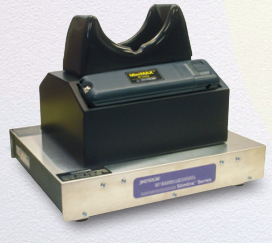

UV-Protective Eye and Face Wear Specially designed to protect users against unwanted UV exposure.

MiniMAXTM UV Viewing Station Space-saving mini-cabinet and UV lamp combination makes the perfect portable UV viewing station. Light weight and compact.

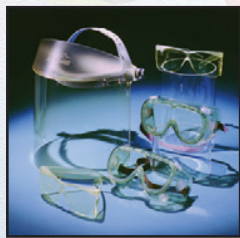
CORPORATION wWW.spectroline.com

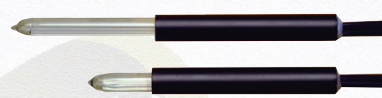

Miniature UV Pencil Lamps Double-bore quartz lamps used for wavelength calibration and emission. Custom lamps for OEM applications also available.

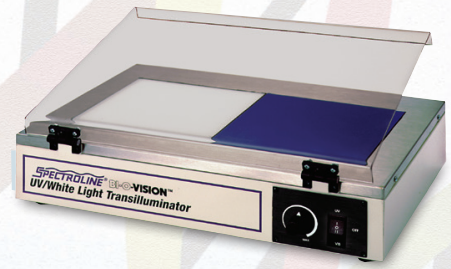

UV Transilluminators

Deliver superior irradiance uniformity and UV intensity for all viewing and photodocumentation needs.

Buy Direct From Us and Save!

Call 1-800-274-8888 (516-333-4840 outside USA) or email us at sales@spectroline.com and mention Code B315

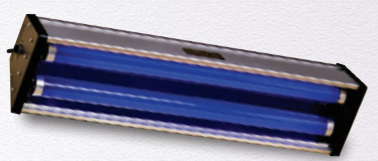

UV Bench and Display Lamps Lamps with various wattages and UV wavelengths provide both high intensity and wide area of light coverage.

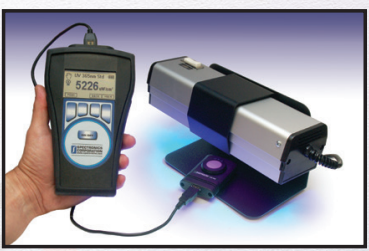

AccuMAX ${ }^{\mathrm{TM}}$ Digital Meters Advanced microprocessor-controlled units with software-driven functions. Ensure maximum accuracy and reliability in measurement of UV and visible light sources.

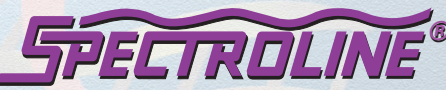

The World Leader in UV Technology! 
A

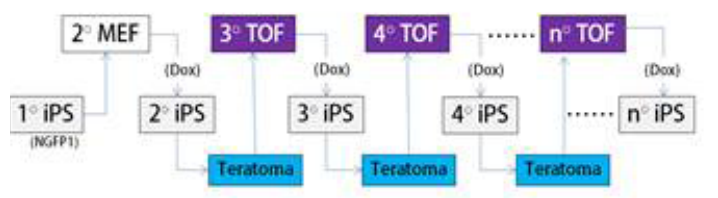

B

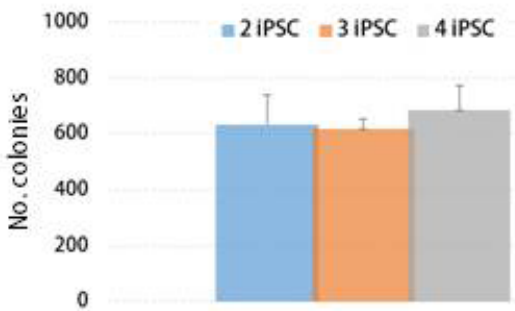

\section{C}

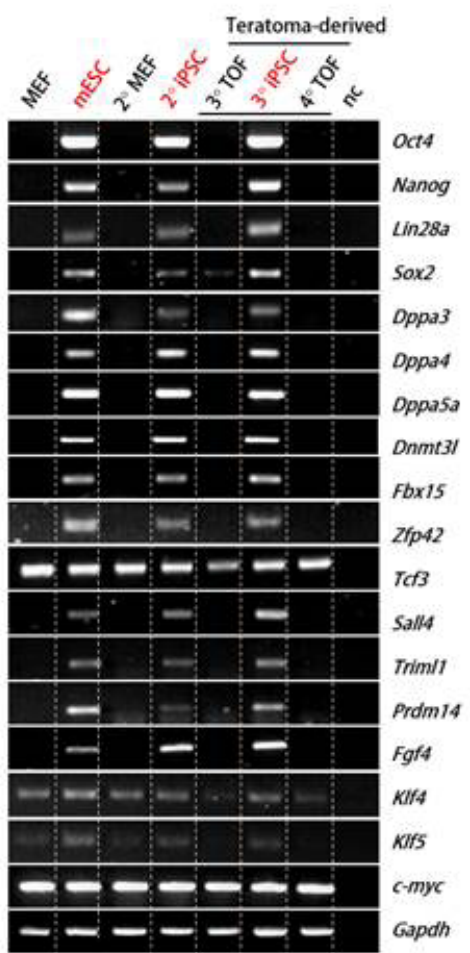

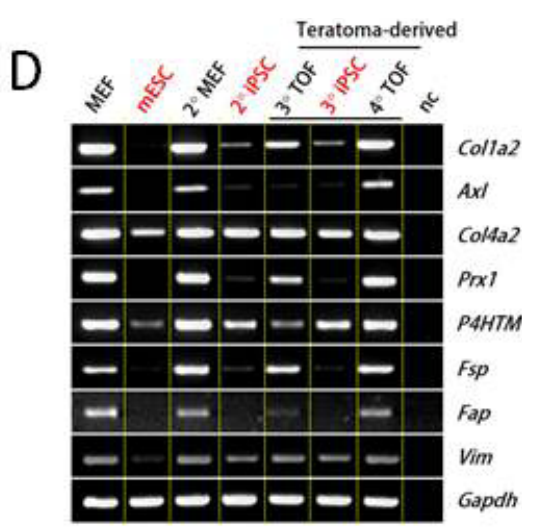

Figure 4. Stable maintenance of cellular features in teratoma-derived fibroblast-like cells (TOFs) and their induced pluripotent stem cells (iPSCs) of different generations. (A) The cyclic procedure of teratoma formation, TOF derivation, and iPSC production. (B) The ability of secondary ( $2^{\circ}$ ) mouse embryonic fibroblast (MEF), tertiary ( $\left.3^{\circ}\right)$ TOF, and quaternary ( $\left.4^{\circ}\right)$ TOF to form secondary iPSC (634 \pm 105$)$, $3^{\circ}$ iPSC (619 \pm 32 ), and $4^{\circ}$ iPSC colonies $(685 \pm 86)$, respectively. No difference among these samples was observed at the $P<0.05$ level, as determined by a one-way ANOVA test. (C,D) Expression profiles of pluripotency genes (C) and fibroblast-specific genes (D) in fibroblasts and iPSCs of different generations. MEF and mouse embryonic stem cells (mESCs) were obtained from C57/B6 fetuses and J1 mESC line, respectively. nc: no-template negative control.

reprogramming system in the genome, secondary "adult" fibroblast cultures such as tail tip fibroblasts (12) could also be established. This protocol is very convenient, and secondary iPSCs can be generated with it in a rather simple manner. However, it has a weak point. It requires transgenic animals harboring certain types of inducible reprogramming systems. Transgenic animals would have to be newly established every time we were faced with the use of new combinations of reprogramming factors or the need for the addition or removal of certain existing factor(s) from the Dox-inducible reprogramming system. Our TOF secondary system can avoid such a cumbersome procedure because it does not need transgenic animals; whatever reprogramming systems we would use for iPSC generation, secondary TOFs could be obtained by simple injection of the resulting iPSCs into NOS-SCID mice to produce teratomas.

After 4 to 5 rounds of division, TOFs slowed their growth and eventually ceased to proliferate further. This indicates a drawback of using TOFs as a source of secondary fibroblasts. However, the ability to obtain a substantial amount of passage-zero (p0) TOFs from a single mass of teratoma may compensate for this shortcoming. Moreover, considering that secondary fibroblasts of earlier passages (usually p2 or p3 cells) have been used for Dox induction, being able to obtain a large amount of early primary cells provides a strong basis for the use of TOFs. Nevertheless, if this low proliferation ability might be an indication of an improper culture condition that affects the nature of the cells, further efforts should be made to establish a more suitable environment for the sustainable culture of TOFs.

To obtain secondary fibroblasts of human origin, an in vitro differentiation strategy has been developed to obtain primary iPSCs carrying Dox-inducible proviral genomes, which are allowed to differentiate into fibroblasts via embryoid body formation $(9,11)$. It has been reported that in vitro-derived fibroblasts are more amenable to reprogramming than are the primary fibroblasts $(5,6)$, and this difference may indicate a lack of exposure to potent differentiation cues in vitro and/or a shift in characteristics due to the adaptation to the in vitro environment. Our TOF secondary system can avoid such criticisms, because it uses in vivo-formed teratomas enriched with in vivo-derived fibroblast-like cells as a source of secondary cells. As far as we know, there has been no report on the production of human secondary iPSC system except for those fibroblast cultures that were directly differentiated in vitro from iPSCs. We are currently attempting to establish human iPSC clones carrying a Dox-inducible secondary system and to derive TOFs from the resulting iPSCs by means of teratoma formation. The inherent advantage of using teratomas is that they would allow for the generation of a secondary reprogrammable system from human cells.

\section{Author contributions}

J.S.P. and S.M.C. executed experiments and contributed to the data collection and analysis. B.M. performed the literature search and contributed to data collection. S.K. performed the RT-PCR analysis. Y.K.K. designed the study, interpreted, and wrote the manuscript. 


\section{Acknowledgments}

This study was supported by grants from the National Research Foundation of Korea (2006-2004082 and 2015R1A2A2A01003117), NRF-SRC program (2011-0030049), Biogreen21, and KRIBB Research Initiative program.

\section{Competing interests}

The authors declare no competing interests.

\section{References}

1. Wernig, M., A. Meissner, R. Foreman, T. Brambrink, M. Ku, K. Hochedlinger, B.E. Bernstein, and R. Jaenisch. 2007. In vitro reprogramming of fibroblasts into a pluripotent ES-cell-like state. Nature 448:318-324.

2. Okita, K., T. Ichisaka, and S. Yamanaka. 2007. Generation of germlinecompetent induced pluripotent stem cells. Nature 448:313-317.

3. Takahashi, K. and S. Yamanaka. 2006. Induction of pluripotent stem cells from mouse embryonic and adult fibroblast cultures by defined factors. Cell 126:663-676.

4. Takahashi, K., K. Tanabe, M. Ohnuki, M. Narita, T. Ichisaka, K. Tomoda, and S. Yamanaka. 2007. Induction of pluripotent stem cells from adult human fibroblasts by defined factors. Cell 131:861-872.

5. Yu, J., M.A. Vodyanik, K. Smuga-Otto, J. Antosiewicz-Bourget, J.L. Frane, S. Tian, J. Nie, G.A. Jonsdottir, et al. 2007. Induced pluripotent stem cell lines derived from human somatic cells. Science 318:1917-1920.

6. Park, I.H., R. Zhao, J.A. West, A. Yabuuchi, H. Huo, T.A. Ince, P.H. Lerou, M.W. Lensch, and G.Q. Daley. 2008. Reprogramming of human somatic cells to pluripotency with defined factors. Nature 451:141-146.

7. Brambrink, T., R. Foreman, G.G. Welstead, C.J. Lengner, M. Wernig, H. Suh, and R. Jaenisch. 2008. Sequential expression of pluripotency markers during direct reprogramming of mouse somatic cells. Cell Stem Cell 2:151-159.

8. Stadtfeld, M., N. Maherali, D.T. Breault, and K. Hochedlinger. 2008. Defining molecular cornerstones during fibroblast to iPS cell reprogramming in mouse. Cell Stem Cell 2:230-240.

9. Maherali, N., T. Ahfeldt, A. Rigamonti, J. Utikal, C. Cowan, and K. Hochedlinger. 2008. A high-efficiency system for the generation and study of human induced pluripotent stem cells. Cell Stem Cell 3:340-345.

10. Wernig, M., C.J. Lengner, J. Hanna, M.A. Lodato, E. Steine, R. Foreman, J. Staerk, S. Markoulaki, and R. Jaenisch. 2008. A druginducible transgenic system for direct reprogramming of multiple somatic cell types. Nat. Biotechnol. 26:916-924.

11. Hockemeyer, D., F. Soldner, E.G. Cook, Q. Gao, M. Mitalipova, and R. Jaenisch. 2008. A drug-inducible system for direct reprogramming of human somatic cells to pluripotency. Cell Stem Cell 3:346-353.

12. Carey, B.W., S. Markoulaki, C. Beard, J. Hanna, and R. Jaenisch. 2010. Single-gene transgenic mouse strains for reprogramming adult somatic cells. Nat. Methods 7:56-59.

13. Kwon, S., J.S. Park, B. Min, and Y.-K. Kang. 2015. Differences in the gene expression profiles of slow- and fast-forming pre-induced pluripotent stem cell colonies. Stem Cells Int. 2015:195928.

14. Cho, S., J.S. Park, and Y.K. Kang. 2013. Regulated nuclear entry of over-expressed Setdb1. Genes Cells. 18:694-703.

15. Cho, S., J.S. Park, S. Kwon, and Y.K. Kang. 2012. Dynamics of Setdb1 expression in early mouse development. Gene expression patterns. Gene Expr Patterns. 12:213-218.

16. Ben-David, U., Q.F. Gan, T. Golan-Lev, P. Arora, O. Yanuka, Y.S. Oren, A. Leikin-Frenkel, M. Graf, et al. 2013. Selective elimination of human pluripotent stem cells by an oleate synthesis inhibitor discovered in a high-throughput screen. Cell Stem Cell 12:167-179.

Received 20 July 2014; accepted 05 May 2015.

Address correspondence to Yong-Kook Kang, 111 Gwahangno, Yuseong-gu, Daejeon, 305-806, Korea. E-mail: ykkang@kribb.re.kr

\section{NEW!} Affinity ITC

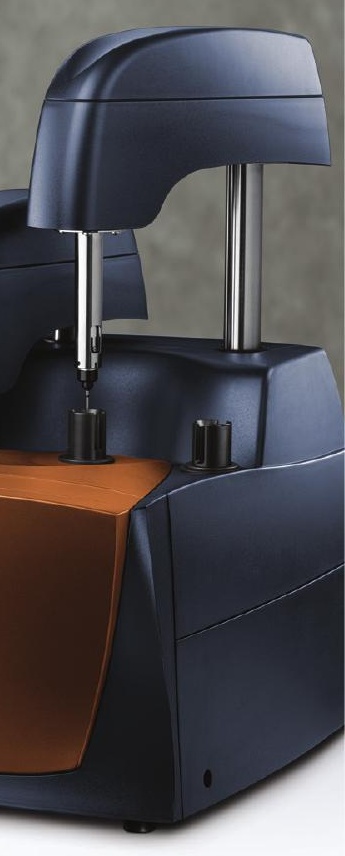

\section{What others have attempted, TA has perfected}

The Affinity ITC is a fully automated, highly sensitive, titration calorimeter for characterizing a wide variety of molecular interactions.

\section{Features:}

- NEW 96-well temperature-controlled liquid handling with Intelligent Hardware Positioning for walk-away automation and precise, reliable injections.

- NEW Flex Spin ${ }^{\mathrm{TM}}$ for the widest range of stirring speeds. Slow speed mixing (up to 10X lower than competitive units) for high sensitivity and to protect delicate structures.

- NEW AccuShot'TM introduces samples at the right location for efficient mixing.

- NEW fully automated and user-selectable system cleaning routines eliminates run-to-run contamination.

- NEW Nano-Analyze software with batch processing for automated data analysis.

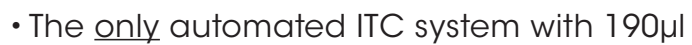
or $1 \mathrm{ml}$ sample cells.

\section{FlexSpin" $\mid$ AccuShot"}

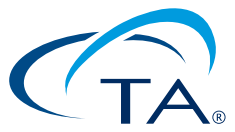

